XLVIth Zakopane School of Physics, International Symposium Breaking Frontiers, Zakopane, Poland, May 16-21, 2011

\title{
Study of the Thermoluminescence of CVD Diamond
}

\author{
A. Karczmarska*, M. Mitura-Nowak, T. Nowak and M. MarszaŁeK \\ The H. Niewodniczański Institute of Nuclear Physics, Polish Academy of Sciences \\ E. Radzikowskiego 152, 31-342 Kraków, Poland
}

\begin{abstract}
This work reports some thermoluminescent properties of chemical vapour deposition diamond film grown by $5 \mathrm{~kW}$ microwave assisted plasma chemical vapour deposition. The $2^{\prime \prime}$ diamond sample with the thickness of $32 \mu \mathrm{m}$ was exposed to gamma radiation in the dose range 0.6-55 Gy. The thermoluminescent glow curve of diamond sample displayed a peak with the maximum centered at $240{ }^{\circ} \mathrm{C}$. The linearity of the detector response in the range of 10-55 Gy was observed. The bleaching effect, seen as fading of the thermoluminescent signal when the device was exposed to light in the visible range, was also noticed. Two-dimensional dose distribution was measured using 2D thermoluminescent reader equipped with a sensitive $640 \times 480$ pixels charge coupled device camera. The surface morphology of the diamond film was observed with scanning electron microscope and atomic force microscope. The grain size was estimated as about $15 \mu \mathrm{m}$. The Raman spectroscopy was used to characterize phase purity. The width and position of the diamond Raman band around $1332 \mathrm{~cm}^{-1}$ indicated that the coating is crystalline and of good quality. It is concluded that good quality chemical vapour deposition diamond coating could be promising material for thermoluminescent dosimetry.
\end{abstract}

PACS: 81.10.Bk, 81.05.ug, 78.60.Kn

\section{Introduction}

Chemical vapour deposition (CVD) diamond is a promising material for numerous applications because of the excellent properties of diamond such as extreme mechanical hardness, broad optical transparency from the deep UV to the far IR region of the electromagnetic spectrum, good electrical insulation and resistance to chemical corrosion $[1,2]$. The potential use of such material for ionizing radiation dosimetry by thermoluminescence have been investigated since 1980. Natural diamond has been examined but thermoluminescence (TL) signal was very weak and not reproducible $[3,4]$. Diamond $(Z=6)$ with its atomic number being close to the effective atomic number of biological tissue $(Z=7.42)$ can be considered as a tissue-equivalent material. Additionally, it is radiation hard and non-toxic [5]. Such properties make CVD diamond suitable for passive dosimetry application in the monitoring of doses around the accelerators used in radiotherapy and in fusion reactors and high energy accelerators.

The aim of this work was investigation of TL response of CVD diamond coating after irradiation with various doses of ionising radiation. The sensitivity to the radiation, linearity of the detector response, bleaching effect, homogeneity of TL response from diamond film and the correlation between structure and TL properties were studied.

\section{Experimental details}

Diamond films were deposited on silicon substrates in a SEKI MWCVD system working at $2.45 \mathrm{GHz}$ with an

\footnotetext{
* corresponding author; e-mail:

agnieszka.karczmarska@ifj.edu.pl
}

ASTEX $5 \mathrm{~kW}$ magnetron plasma source. Prior to the deposition, commercial single-crystal $\mathrm{Si}$ (111) wafer was ultrasonically treated in mixture of ethanol with diamond powder with a grain size of $40 \mu \mathrm{m}$. The substrate was mounted on a water-cooled Mo holder and heated by the plasma. Before each experimental run the vacuum system was pumped down by a turbomolecular pump up to $2 \times 10^{-6}$ Torr. The substrate temperature was measured by a two-color pyrometer (Williamson Corp., 450$1400^{\circ} \mathrm{C}$ ) focusing on the central part of the substrate, at a distance of $35 \mathrm{~cm}$. Before introduction of methane into the deposition chamber, the silicon substrate was pre-treated in the hydrogen plasma for $4 \mathrm{~min}$. The growth parameters were: microwave power of $4.9 \mathrm{~kW}$, pressure of 125 Torr, and $\mathrm{H}_{2} / \mathrm{CH}_{4} / \mathrm{O}_{2}$ flow of $478 / 20 / 2 \mathrm{sccm}$, respectively. Deposition was conducted for $12 \mathrm{~h}$ to get diamond film with thickness of $32 \mu \mathrm{m}$. The deposition rate calculated from the deposited mass and the total deposition time equals $2.6 \mu \mathrm{m} / \mathrm{h}$.

Optical microscopy (OM), atomic force microscopy (AFM) and scanning electron microscopy (SEM) were used to observe the morphology of diamond film. Micro-Raman spectra of the sample were taken using Nicolet Almega Raman spectrometer with $532 \mathrm{~nm}$ laser operating at beam power of $25 \mathrm{~mW}$ on a $0.7 \mu \mathrm{m}$ diameter spot size.

The diamond film was exposed to gamma radiation using a Theratron $780 \mathrm{E}{ }^{60} \mathrm{Co}$ radiotherapy unit, with reference dosimetry provided by a Farmer ionization chamber. The gamma irradiation of diamond was performed in the $0.6-55$ Gy dose range at $0.65 \mathrm{~Gy} / \mathrm{min}$ dose rate. The sample was irradiated under a $5 \mathrm{~mm}$ thick PMMA build-up plate. TL measurements were performed $10 \mathrm{~min}$ after irradiation. Two-dimensional dose distribution measurements were done by using a large-area TL reader with a heater of diameter of $60 \mathrm{~mm}$ and equipped with a 12-bit CCD camera of $640 \times 480$ pixels resolution. This camera 
registered up to 4500 counts in one pixel. The heating rate of the reader was $2.8^{\circ} \mathrm{C} / \mathrm{s}$. The sample was heated up to maximum temperature of $330^{\circ} \mathrm{C}$.

\section{Results and discussion}

The glow curves of investigated diamond sample presenting TL signal as a function of gamma irradiation dose are shown in Fig. 1. The sample was irradiated by gamma rays in the $0.6-55$ Gy dose range, but the TL response was observed from 9 Gy. The diamond exhibited glow curve with a maximum of amplitude at $230^{\circ} \mathrm{C}$ situated in the same position for all applied doses (Fig. 1). Small shoulder was observed around $130^{\circ} \mathrm{C}$ but only for the highest doses. Gastélum et al. [6] observed, in MWCVD diamond samples with gas composition of $\mathrm{H}_{2}, \mathrm{CH}_{4}$ and additional $\mathrm{CO}$, a single, well-resolved TL glow peak, which shifted towards low temperature values as the dose increased. In our case, TL glow curve maintains the single well-resolved peak independently of the irradiation dose, and is better qualified for higher doses.

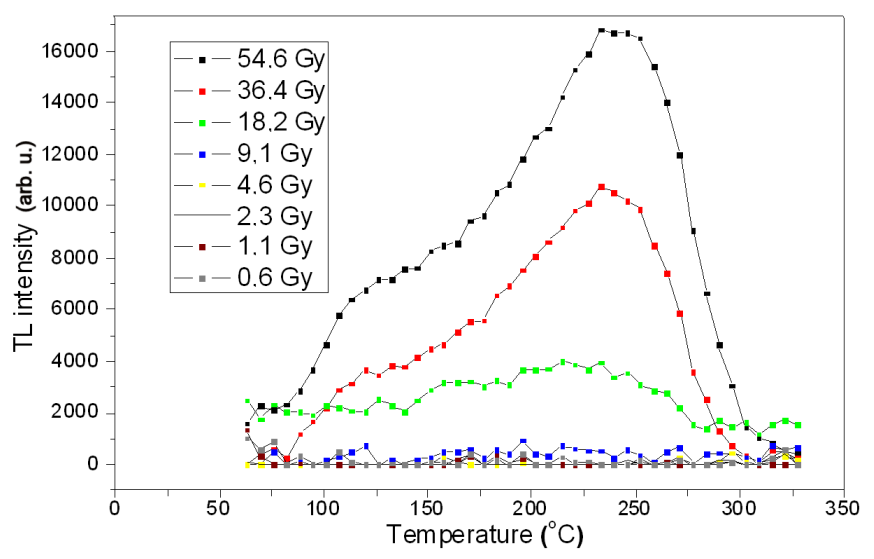

Fig. 1. Thermoluminescence (TL) glow curve of CVD diamond films exposed to 0.6-55 Gy gamma irradiation doses.

One of the important features of the detectors used for dosimetry is the response linearity, i.e. the range where the measured signal remains proportional to the applied dose $[7,8]$. The TL signal as a function of dose shown in Fig. 2 exhibits linearity from 10 to 55 Gy. The TL response at doses lower than 10 Gy was below the detection limit.

Strong limitation of diamond use in TL dosimetry is the possible release of trapped carrier between irradiation and measurement due to daylight $[9,10]$. The testing of these properties was conducted on diamond sample after gamma irradiation. The sample was irradiated with the dose of $36.4 \mathrm{~Gy}$ at room temperature in darkness, and then it was exposed to the daylight for $10 \mathrm{~min}$. As expected, the observed TL signal vanished after such illumination (Fig. 3).

In the literature there are a few works reporting gamma irradiation of diamond samples. Gastéluma and

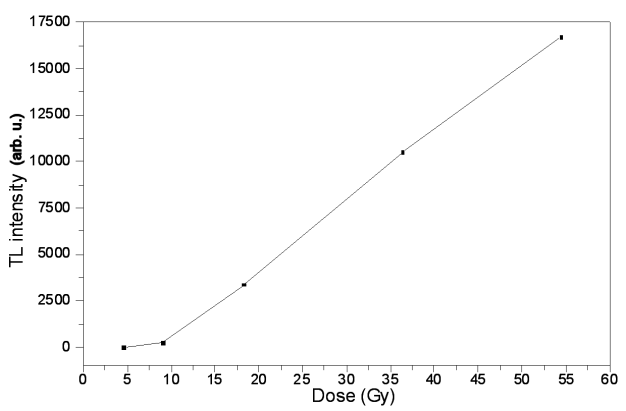

Fig. 2. TL signal plotted as a function of the gamma irradiation dose for CVD diamond sample.

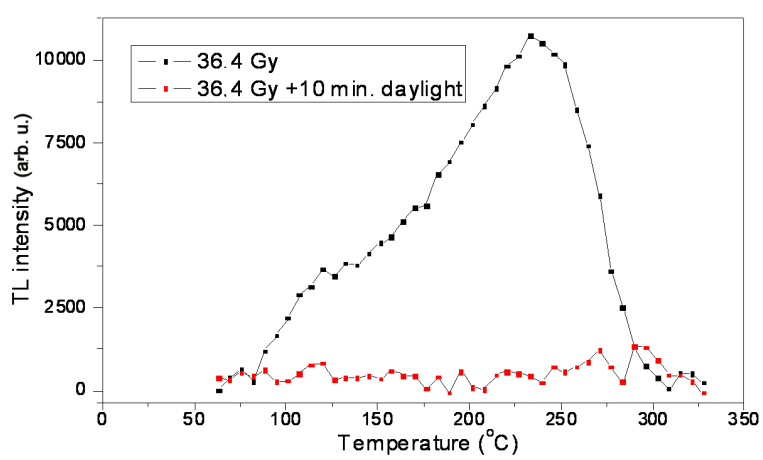

Fig. 3. The behaviour of TL peak as a function of TL intensity of bleaching light in the case of dose of $36.4 \mathrm{~Gy}$.

Cruz-Zaragoza [11] reported that the diamond films exhibited 3-5\% TL reproducibility independent of the irradiation dose rate, which is comparable to commercial dosimeter materials. In [12] samples grown by the hot filament CVD method with thicknesses of 180 and $500 \mu \mathrm{m}$ showing a linear dose behavior in the 1-50 Gy range were presented. Two samples with thicknesses of 6 and $12 \mu \mathrm{m}$ prepared by MW CVD were studied in [6]. The film with thickness of $12 \mu \mathrm{m}$ showed a lower TL efficiency as compared to the specimen with thickness of $6 \mu \mathrm{m}$. The Raman spectroscopy and SEM analyses indicated that special care and control of growing conditions parameters should be taken to guarantee high-quality nucleation of $s p^{3}$ diamond bonding. Barboza-Flores et al. [13] investigated the TL properties of undoped and nitrogen-doped CVD diamond grown on (001) silicon substrate. The results obtained by Benabdesselam et al. [8] showed that the CVD diamond has one glow peak at $540 \mathrm{~K}$. TL properties of CVD diamond film were compared with those of commercial LiF TLDs.

A few works report the homogeneity of response from large-area diamond basing on TL studies [14]. Benabdesselam et al. [15] reported samples cut from free-standing polycrystalline CVD diamond wafer, synthesized at large scale and exposed to radiation which showed the same features everywhere in the diamond wafer. At IFJ in Kraków a novel method of determination of two-dimensional dose distribution of CVD dia- 


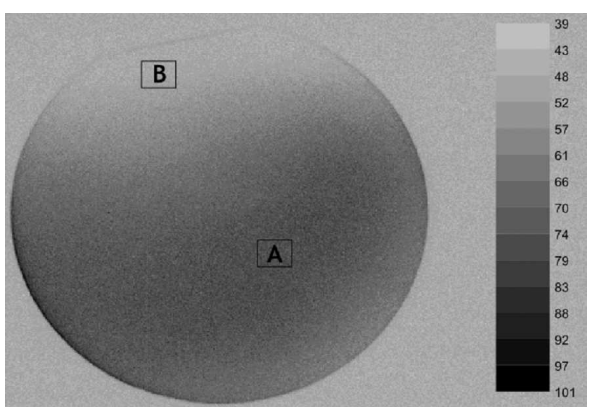

Fig. 4. Thermoluminescence output from readout of CVD diamond detector after irradiating of 36.4 Gy of Co-60.

mond wafers of diameter up to $6 \mathrm{~cm}$ was developed using 2D TL reader equipement. The CVD diamond studied with this method exhibited satisfactory sensitivity and high homogeneity [16].

Figure 4 presents the integrated TL light output of CVD diamond coating, where the brightness of separated parts of the detector represents the intensity of its TL signal. Visible non-homogeneity of light emission within individual parts, come from their non-uniform internal structure. In order to analyze the differences in homogeneity of the diamond coating, the layer was measured in two places (marked in Fig. 4, place A and B). The strong TL efficiency differences observed in the image of diamond surface were confirmed through the examination of the Raman spectra.

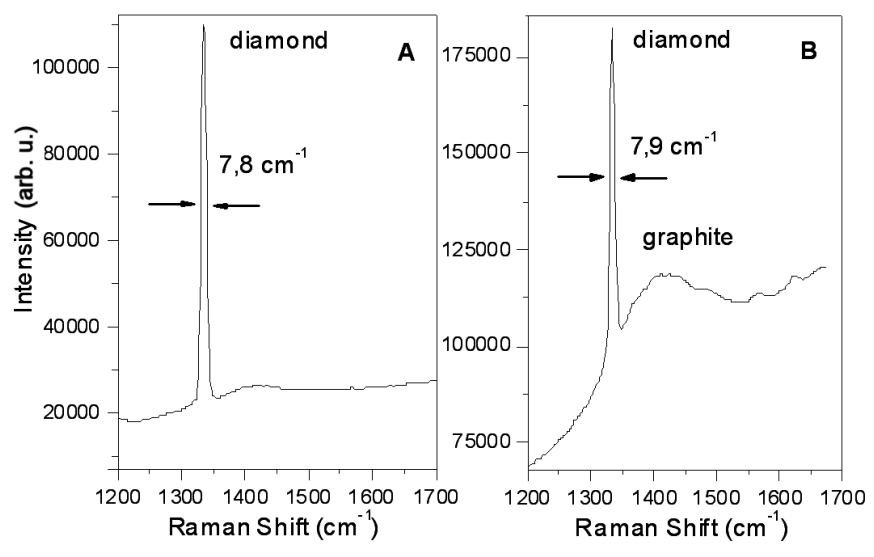

Fig. 5. Raman spectra of polycrystalline diamond coating taken from two places marked in Fig. 4 as A and $\mathrm{B}$.

The Raman spectroscopy is most extensively used to characterize CVD diamonds because of its ability to distinguish between different forms of carbon. The penetrating depth of the laser into the sample depends strongly on the film quality [1, 17]. In the Raman spectrometers a laser beam of a certain wavelength can be focused at a spot of about $1 \mu \mathrm{m}$. In CVD films, the presence of diamond is represented by a sharp line at
$1332 \mathrm{~cm}^{-1}$ which is due to a vibration of the two interpenetrating cubic sub-lattices of diamond.

The graphite usually gives rise to two broader peaks around 1335 and $1580 \mathrm{~cm}^{-1}$ called D (disordered graphite) and $\mathrm{G}$ (crystalline graphite), respectively. The ratio of the intensities of the diamond peak to the graphite peak indicates how much of each phase is present, but it is also dependent on the wavelength of excitation. It should be remembered that in the case of visible light the cross-section of the Raman scattering for the $s p^{2}$ bonds is much greater than for the $s p^{3}$ bonds, and therefore the presence of even a small amount of $s p^{2}$ bonds in the diamond layer can significantly contribute to the Raman spectrum [18].

Figure 5 shows the micro-Raman spectra of the sample excited by a $532 \mathrm{~nm}$ green laser. The occurrence in area $\mathrm{A}$ of the first-order diamond line around $1334 \mathrm{~cm}^{-1}$ proves the diamond nature of this sample. The linear background and lack of contribution from scattering by the non-diamond phase was observed. The full width at half maximum (FWHM) of the diamond Raman peak in the visible Raman spectra of the sample is about $7.8 \mathrm{~cm}^{-1}$. Comparing FWHMs of the films with that of IIa natural diamond $\left(2.2 \mathrm{~cm}^{-1}\right)$, used for calibration of the Raman system, and comparing with other polycrystalline samples presented for example by Tang [19], it is clear that this area is high quality diamond layer. The area B in Fig. 5 shows the presence of a well defined highly ordered diamond ( $s p^{3}$ bonding) at $1334 \mathrm{~cm}^{-1}$, together with the graphite band at $1400 \mathrm{~cm}^{-1}$. Diamond, graphite or some other form of carbon bound to the silicon substrate significantly affect the shape of the TL glow curve.

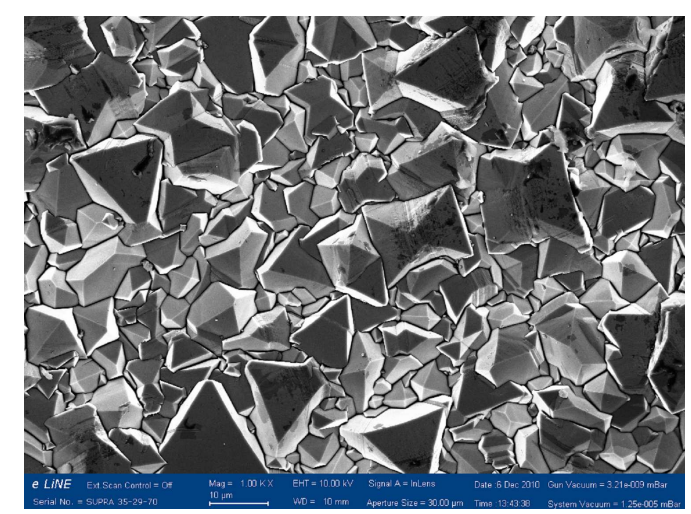

Fig. 6. SEM micrographs of polycrystalline diamond coating taken from place marked in Fig. 4 as A.

Information on the nucleation process of MWCVD diamond is also obtained from SEM micrographs of the surface morphology. In Fig. 6 the SEM micrographs of the sample taken in place $\mathrm{A}$ is shown. It demonstrates that the coating is dense and well crystallized and clearly displays the formation of triangular (111) facets of crystals with $15 \mu \mathrm{m}$ grain size. We also received similar results by the AFM. 


\section{Conclusions}

For many years, diamond has been considered as material with great potential in radiation dosimetry applications. The reason why diamonds were not widely used in this area is primarily the price of good quality natural diamond. Due to development of a chemical vapour deposition method, the production of cheap diamond crystals of a good quality is now possible. Synthetic diamond samples show a TL efficiency comparable to commercial LiF TLD-100 dosimeters. The quality and homogeneity of CVD diamond layers depend strongly on growth parameters and even minor changes of the structural defects in the growing coating CVD, may significantly affect the local variations of the TL response of CVD diamond. This matter requires systematic study of the basic parameters of the deposition, such as gas composition, substrate temperature and microwave power, in order to produce optimal diamond samples for use as a dosimeter material.

$2 \mathrm{D}$ thermoluminescence appears to be a perfect tool for testing the homogeneity and the purity of diamond samples. The results of this work indicate that the TL peak temperature at the maximum intensity for sample which was gamma irradiated is situated in the same position for all doses. TL signal as a function of dose demonstrates the linearity in the range from 10 to 55 Gy. The Raman spectroscopy and SEM analyses indicate that the structure of the coating is not the same over all sample area. The occurrence of the line around $1334 \mathrm{~cm}^{-1}$ in the Raman spectra at area A proves the diamond nature of this sample. However, the presence of $s p^{2}$ bonds at area $\mathrm{B}$ causes the need of further research on the optimization of the deposition process.

In this work, the unique thermoluminescent properties of CVD diamond i.e. linear dose response in a wide radiation dose ranges, was demonstrated. We also observed strong limitation of diamond use in TL dosimetry related to vanishing of TL signal between irradiation and measurement due to daylight. Therefore, further studies of structure and thermoluminescence together with the optical and thermal fading investigation are needed.

\section{Acknowledgments}

Agnieszka Karczmarska has been partly supported by the Ph.D. scholarship in the framework of the EU Human Capital Operation Program and the DOCTUS scholarship cofounded by European Social Fund.

\section{References}

[1] J.J. Gracio, Q.H. Fan, J.C. Madaleno, J. Phys. D, Appl. Phys. 43, 374017 (2010).

[2] L.K. Bigelow, M.P. D'Evelyn, Surf. Sci. 500, 986 (2002).

[3] E.A. Burgemeister, Phys. Med. Biol. 26, 269 (1981).

[4] B. Planskoy, Phys. Med. Biol. 25, 519 (1980).

[5] P. Olko, Ł. Czopyk, M. Kłosowski, M.P.R. Waligórski, Radiat. Meas. 43, 864 (2008).

[6] S. Gastélum, E. Cruz-Zaragoza, V. Chernov, R. Meléndrez, M. Pedroza-Montero, M. Barboza-Flores, Nucl. Instrum. Methods Phys. Res. B 260, 592 (2007).

[7] M. Rebisz, M.J. Guerrero, D. Tromson, M. Pomorski, B. Marczewska, M. Nesladek, P. Bergonzoa, Diam. Relat. Mater. 13, 796 (2004).

[8] M. Benabdesselam, P. Iacconi, D. Briand, J.E. Butler, Diam. Relat. Mater. 9, 1013 (2000).

[9] C. Manfredotti, E. Vittone, F. Fizzotti, A. Lo Giudice, C. Paolini, Diam. Relat. Mater. 11, 446 (2002).

[10] E. Vittone, C. Manfredotti, F. Fizzotti, A. Lo Giudice, P. Polesello, V. Ralchenko, Diam. Relat. Mater. 8, 1234 (1999).

[11] S. Gastélum, E. Cruz-Zaragoza, A. Favalli, R. Meléndrez, V. Chernov, M. Barboza-Flores, Diam. Relat. Mater. 17, 1283 (2008).

[12] S. Gastélum, E. Cruz-Zaragoza, R. Meléndrez, V. Chernov, M. Barboza-Flores, Nucl. Instrum. Meth. Phys. Res. B 248, 103 (2006).

[13] M. Barboza-Flores, S. Gastélum, E. Cruz-Zaragoza, R. Meléndrez, V. Chernov, M. Pedroza-Montero, A. Favalli, Radiat. Meas. 43, 379 (2008).

[14] B. Marczewska, P. Bilski, P. Olko, M.P.R. Waligórski, Radiat. Meas. 38, 833 (2004).

[15] M. Benabdesselam, P. Iacconi, J.E. Butler, J.M. Nigoul, Diam. Relat. Mater. 12, 1750 (2003).

[16] B. Marczewska, P. Bilski, P. Olko, M. Nesladek, P. Bergonzo, M. Rębisz, M.P.R. Waligorski, Phys. Status. Solidi A 199, 119 (2003).

[17] S. Sciortino, Riv. Nuovo Cim. 22, 10 (1999).

[18] J. Filik, Spectrosc. Europe 17, 5 (2005).

[19] C.J. Tang, A.J. Neves, A.J.S. Fernandes, Diam. Relat. Mater. 12, 1488 (2003). 